\title{
Induction of cytomegalovirus-infected labyrinthitis in newborn mice by lipopolysaccharide: a model for hearing loss in congenital CMV infection
}

\author{
$\mathrm{Li} \mathrm{Li}^{1}$, Isao Kosugi ${ }^{1}$, Gui-Ping Han ${ }^{1}$, Hideya Kawasaki ${ }^{1}$, Yoshifumi Arai ${ }^{1}$, Tamotsu Takeshita ${ }^{2}$ and Yoshihiro Tsutsui ${ }^{1}$
}

Congenital cytomegalovirus (CMV) infection is the most common infectious cause of sensorineural hearing loss in children. Here, we established an experimental model of hearing loss after systemic infection with murine CMV (MCMV) in newborn mice. Although almost no viral infection was observed in the inner ears and brains by intraperitoneal (i.p.) infection with MCMV in newborn mice, infection in these regions was induced in combination with intracerebral (i.c.) injection of bacterial lipopolysaccharide (LPS). The susceptibility of the inner ears was higher than that of the brains in terms of viral titer per unit weight. In the labyrinths, the viral infection was associated with the mesenchymal vessels and accompanied by inflammatory cells induced by LPS, causing hematogenous targets of infection in the labyrinths. Viral infection also spread in the perilymph regions such as the scala tympani and scala vestibuli, probably from infected brains via meningogenic and cochlear nerve routes. Viral infection was not observed in the scala media in the endolymph, including the Corti organ. However, viral infection was observed in the spiral limbus, including the stria vascularis. These results suggest that hearing loss caused by labyrinthitis after congenital CMV infection may be enhanced by inflammation caused by systemic bacterial infection in the neonatal period.

Laboratory Investigation (2008) 88, 722-730; doi:10.1038/labinvest.2008.39; published online 12 May 2008

KEYWORDS: animal model; cytomegalovirus; hearing loss; labyrinthitis; lipopolysaccharide; LPS

Cytomegalovirus (CMV), a member of the herpes virus group, is the most significant infectious cause of developmental disorders induced by intrauterine infection in humans, with an average incidence of $1 \%$ of all live births. ${ }^{1,2}$ It is estimated that approximately $5-10 \%$ of infected infants have symptomatic congenital CMV infection at birth, such as microcephaly, periventricular calcification, and microphthalmia. ${ }^{3,4}$ Another $10 \%$ of infected infants have asymptomatic congenital infection, and will subsequently suffer from brain disorders, including mental retardation, sensorineural hearing loss (SNHL), visual disorders, seizures, and epilepsy. ${ }^{5,6}$ Since SNHL is a frequent manifestation among the neurological disorders induced by CMV, the inner ear is considered to be an important target organ after congenital CMV infection. ${ }^{7,8}$ It has been reported that approximately $22-65 \%$ of symptomatic and $6-23 \%$ of asymptomatic children will have hearing loss following congenital CMV infection. 9 Among children with congenital hearing loss,
15-25\% of cases have been estimated to be due to congenital CMV infection. ${ }^{10-12}$ Therefore, the inner ear is considered to be an important target organ in congenital CMV infection.

Experimental models of labyrinthitis as a cause of hearing loss have been reported mainly in adult guinea-pig using guinea-pigs CMV (GPCMV). ${ }^{13-16}$ There was only one report that described the infection of inner ears in fetuses after transplacental infection with GPCMV, in which the infection extended to the perilymph of the inner ears. ${ }^{17}$ It was reported that labyrinthitis in the inner ear was induced in newborn mice after intracranial infection with murine CMV (MCMV). ${ }^{18}$ However, why the inner ears are so susceptible to CMV infection after congenital CMV infection still remains unclear. We have developed mouse models for investigation of the brain abnormalities due to congenital CMV infection. $^{19,20}$

In the present study, we demonstrated the enhanced susceptibility of the inner ear to intraperitoneal (i.p.) infection

\footnotetext{
${ }^{1}$ Department of Pathology, Hamamatsu University School of Medicine, Hamamatsu, Japan and ${ }^{2}$ Department of Otorhinolaryngology, Hamamatsu University School of Medicine, Hamamatsu, Japan

Correspondence: Professor Y Tsutsui, MD, PhD, Department of Pathology, Hamamatsu University School of Medicine, 1-20-1 Handayama, Higashi-ku, Hamamatsu, Shizuoka 431-3192, Japan.

E-mail: ytsutsui@hama-med.ac.jp

Received 02 November 2007; revised 27 February 2008; accepted 07 March 2008
} 
with MCMV in newborn mice in combination with intracerebral (i.c.) injection of bacterial lipopolysaccharide (LPS). We investigated the pathogenesis of MCMV-induced labyrinthitis in the inner ears in comparison with brain infection in this model. We propose a hypothesis in which the susceptibility of the inner ears to CMV infection is increased by inflammation caused by bacterial infection in the neonatal period after congenital CMV infection.

\section{MATERIALS AND METHODS Virus and Cell Cultures}

The Smith strain of MCMV was passaged in mouse embryonic fibroblasts (MEFs). The MEFs were prepared from 12-day-old embryos of ICR mice as reported previously ${ }^{21}$ and were grown in Dulbecco's modified Eagle's minimal essential medium supplemented with penicillin (100 U), streptomycin $(50 \mu \mathrm{g} / \mathrm{ml})$, and $10 \%$ fetal calf serum. The virus was quantified by the plaque assay method of Wentworth and French $^{22}$ with MEF. The titer of the virus stock was $1 \times 10^{8}$ plaque-forming units $(\mathrm{PFU}) / \mathrm{ml}$.

\section{Intraperitoneal Infection}

Pregnant BALB/c mice (16 days of gestation) were obtained from SLC Japan (Hamamatsu, Japan). Newborn mice 2 days after birth were subjected to i.p. infection with MCMV $\left(1 \times 10^{5} \mathrm{PFU}\right)$ in $50 \mu \mathrm{l}$ of minimum essential medium (MEM). At 1 day before the infection, $5 \mu \mathrm{g}$ of LPS from Escherichia coli strain O111:B4 (Sigma, St Louis, MO, USA) in $5 \mu \mathrm{l}$ of PBS was injected into the right cerebral hemisphere of neonatal mice under cryoanesthesia using a 10- $\mu \mathrm{l}$ Hamilton syringe with a 27-gauge needle from the midpoint between the ear and eye. At 1 day after MCMV infection, the same dose of LPS was injected into the left cerebral hemisphere and these alternative-side injections were repeated every 2 days for a total of three times. In other groups, only one injection of $5 \mu \mathrm{g}$ LPS in $5 \mu \mathrm{l}$ PBS was given 1 day before the infection. For disruption of the blood-brain barrier (BBB), mannitol (Sigma), $5 \mu \mathrm{l} / \mathrm{g}$ body weight of $25 \%$ stock solution $^{23}$ was injected four times in the same manner as LPS. Control mice were injected with PBS four times in the same way. At 6 days after the infection, the brains and temporal bones, including the inner ears, were separately taken out under ether anesthesia. For histological analysis, mice were perfused with $4 \%$ paraformaldehyde and fixed for $24 \mathrm{~h}$. After fixation, the brains were cut into three coronal sections, divided equally between the tip and confluens sinuum of the cerebrum. The temporal tissues were decalcified with $10 \%$ tetrasodium EDTA in PBS ( $\mathrm{pH}$ 7.4) for 1 week. The brains and temporal bone tissues were dehydrated and embedded in paraffin. The temporal bone tissues were serially sectioned to expose the whole cross section of the inner ears.

\section{Intracerebral Infection}

At 2 days after birth, newborn mice were subjected to i.c. infection with $1 \times 10^{4} \mathrm{PFU}$ of MCMV in $5 \mu \mathrm{l}$ of MEM into the right cerebral hemisphere at the midpoint between the ear and eye. ${ }^{24}$ LPS was not administered in the i.c.-infected mice. At 6 days after infection, the mice were killed under ether anesthesia. The brains and temporal bone tissues, including the inner ears were prepared for histological analysis in the same way as described above.

All animal procedures were approved by the Animal Care and Use Committee, and were conducted according to the 'the Guideline for Animal Experiments of the Hamamatsu University School of Medicine'.

\section{Isolation of Unfixed Inner Ears}

Temporal bones from the i.p.-infected mice with i.c. injection of LPS or PBS were cut by a hard scissor under the stereo microscope and the inner ear tissues were taken out with a forceps and the weights were measured under sterilized condition and stored at $-80^{\circ} \mathrm{C}$.

\section{Immunohistochemistry}

After deparaffinization and dehydration, sections were treated with citrate buffer (Mitsubishi Chemical Co., Tokyo, Japan) by autoclaving at $121^{\circ} \mathrm{C}$ for $15 \mathrm{~min}$. The sections were incubated with normal goat serum for $10 \mathrm{~min}$ at room temperature. Then immunohistochemical staining was performed using primary antibodies including rat monoclonal antibody $(\mathrm{mAb})$ against MCMV immediate-early-1 (IE1) antigen ${ }^{25}$ or mouse mAb against MCMV IE1 provided by Dr S Jonjic (University of Rijeka, Rijeka, Croatia). We also used rat mAb specific to MCMV early cytoplasmic antigen $(\mathrm{Q} 3 \mathrm{mAb}) \cdot{ }^{24}$ For cellular markers, we used rabbit polyclonal antibody (pAb) specific to factor VIII (von Willebrand factor) (Dako A/S, Denmark), rabbit pAb specific to myeloperoxidase (MPO) (Novocastra, Newcastle, UK) and mouse mAb specific to neuronal nuclei (NeuN) (Chemicon, Temecula, CA, USA). The sections were then sequentially incubated with goat anti-rat or anti-mouse IgG, or goat anti-rabbit IgG conjugated with horseradish peroxidase (Nichirei Biosciences, Japan), and colored with 3,3'-diaminobenzidine (DAB) or 3-amino-9ethylcarbazole (AEC) (Dako), or with goat anti-rabbit or mouse IgG conjugated with alkaline phosphatase (ALP) (Nichirei Biosciences), and colored with Fast Blue BB salt (Sigma) in the presence of naphthol AS-MX phosphate (Sigma). For immunohistochemical double staining of IE1 antigen and MPO, the autoclaved sections were reacted with IE1 mAb, incubated with the secondary antibody, and colored with AEC, and then the sections were reacted with $\mathrm{pAb}$ to MPO, incubated with ALP-labeled secondary antibody, and colored with Fast Blue. For the double staining of IE1 antigen and endothelial marker, the autoclaved sections were reacted with anti-IE1 $\mathrm{mAb}$, incubated with the secondary antibody, and colored with AEC, and then sections were treated with proteinase K (Ready-to-Use) (Dako), reacted with $\mathrm{pAb}$ to factor VIII, incubated with ALP-labeled secondary 
Ab, and colored with Fast Blue. For the double staining of viral antigen and neuronal marker, the autoclaved sections were reacted with $\mathrm{Q} 3 \mathrm{mAb}$, incubated with the secondary antibody, and colored with AEC, and then the sections were reacted with NeuN mAb, incubated with ALP-labeled secondary antibody, and colored with Fast Blue.

\section{Auditory Brainstem Response}

Auditory brainstem response (ABR) was assessed as described previously. ${ }^{17}$ Mock- or MCMV-i.p.-infected mice with i.c. injection of PBS or LPS were used 4 weeks after infection. Needle electrodes were placed subcutaneously under anesthesia with sodium pentobarbital $(120 \mathrm{mg} / \mathrm{kg}$, i.p.). The reference electrode was inserted beneath the pinna of the measured ear, the ground beneath the skin of the upper part of the nose, and the active electrode beneath the skin on the top of the head. Responses for 1024 sweeps were averaged at each intensity level near the threshold, in 5-dB sound pressure level (SPL) steps. The threshold was defined as the lowest intensity level at which a clear waveform was visible in the evoked trace and was determined by visual inspection of the responses.

\section{RESULTS}

\section{Enhancement of Viral Replication of the Inner Ear in MCMV-i.p.-Infected Mice by i.c. Injection of LPS}

For systemic infection, newborn mice were infected by i.p. inoculation with MCMV. However, only small numbers of viral antigen-positive cells were observed in the brains, and almost no viral antigen-positive cells were observed in the inner ears. Therefore, newborn mice infected by i.p. inoculation were i.c. injected one or four times with LPS (Figure 1). As for survival rate, more than $93 \%$ of the i.p.infected mice with i.p. injection of PBS survived. However, only $55 \%$ of MCMV-i.p.-infected mice that were injected four times with LPS survived and $64 \%$ of mock-infected mice that were injected four times with LPS survived. In the mice with one time injection of LPS, $62 \%$ of infected mice survived, while the survival rate of mock-infected mice was $91 \%$ (Figure 1). Survival rate of MCMV i.p.-infected mice that were injected four times with i.c. injection of mannitol was 93\% (1 died out of 16 mice). Plaque assays showed that the viral titers in both the inner ears and brains were markedly increased in the i.p.-infected mice that were injected four times with LPS as compared with those in i.p.-infected mice with PBS (Figure 2). In the mice with one time injection of LPS, viral titers were not significantly increased in the inner ears, although the viral titers of the brains were moderately increased. In the i.p.-infected mice that were injected four times with LPS, the viral titers per milligram of the inner ears were significantly higher than viral titers per milligram of the brains (Figure 2).

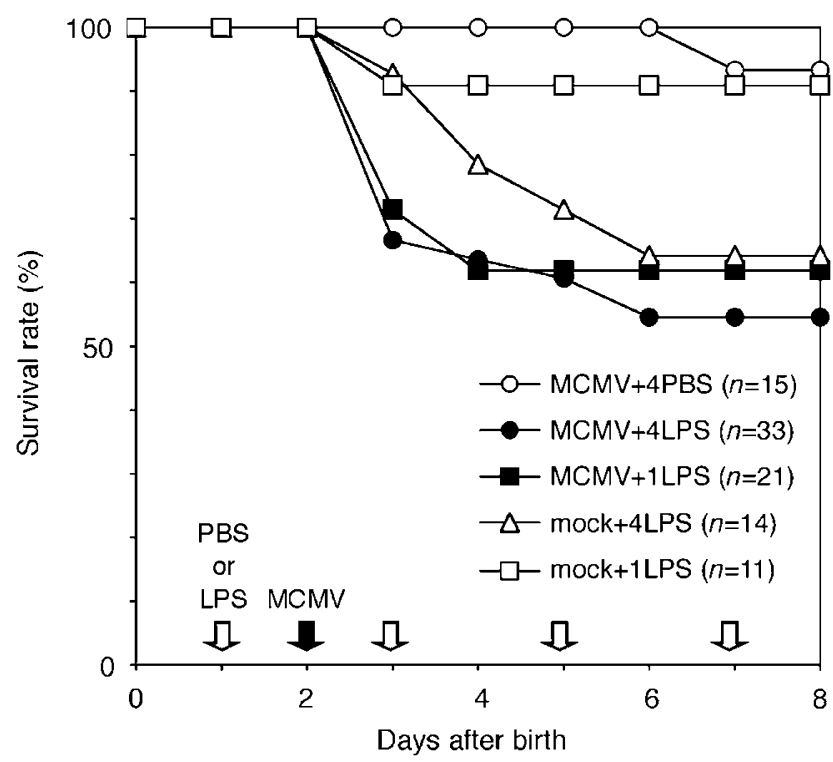

Figure 1 Survival rate of newborn mice i.p. infected with MCMV in combination with i.c. injections of LPS or PBS. Newborn BALB/c mice were i.p. inoculated with MCMV $\left(1 \times 10^{5}\right.$ PFU) 2 days after birth. At 1 day before the infection, LPS $(5 \mu \mathrm{g} / 5 \mu \mathrm{l})$ was injected into the bilateral brains. After MCMV infection, the same dose of LPS was injected into the other side of the brains alternatively three times or left without additional injection of LPS. Control mice were injected with PBS in the same way after i.p. infection with MCMV. Survival rates of mock-infected newborn mice that were injected one or four times with LPS are also shown.

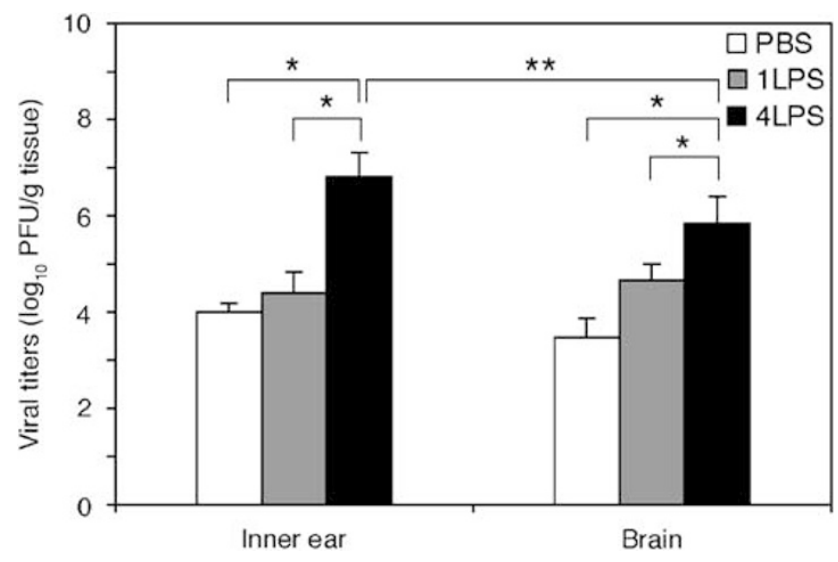

Figure 2 Viral titers of the inner ears and the brains in mice i.p. infected with MCMV and one or four times i.c. injections of LPS or PBS. The inner ear tissues and brains were excised 6 days after infection and weighed and stored at $-80^{\circ} \mathrm{C}$. The tissues were homogenized in $500 \mu \mathrm{l}$ of MEM. A part of the supernatant from each sample was subjected to a plaque assay. Viral titers were expressed as PFU per milligram of organ. ${ }^{\star} P<0.01$ by Student's $t$-test. ${ }^{*} P<0.05$ by Student's $t$-test.

Induction and Distribution of Virus-Infected Cells in the Labyrinths and Brains in i.p.-Infected Mice with LPS In the MCMV-i.p.-infected mice with i.c. injection of PBS, only small numbers of virus-infected cells were observed in the brains, and no infected cells were observed in the inner 
ears in more than 15 mice. In MCMV-i.p.-infected mice with i.c. injection of mannitol, no viral antigen-positive cells were observed in the inner ears. In the MCMV-i.p.-infected mice that were injected four times with LPS, IE1-positive cells were frequently observed in sections of both the inner ears and brains. In the MCMV-i.p.-infected mice with one time injection of LPS, viral antigen-positive cells in the inner ears were observed in only one mouse out of the 12 infected mice $(8.3 \%)$.

In the labyrinths of the inner ears, virus-infected cells were observed in about $75 \%$ of the i.p.-infected mice that were injected four times with LPS. Virus-infected cells stained with anti-IE mAb were observed in the mesenchymal $(\mathrm{M})$ region of labyrinths, cochlear nerve $(\mathrm{CN})$ regions, and in perilymph regions including the scala tympani (ST) and scala vestibuli (SV), but not in the endolymph (Figure 3 ), although the numbers of virus-infected cells were markedly different among the infected mice (Figure 4a). Infected cells were most frequently observed in the mesenchymal regions, including the spiral ganglia (SG) (Figure $3 \mathrm{~b}$ and $\mathrm{c}$ ), while moderate numbers of virus-infected cells were also observed along the $\mathrm{CN}$ regions (Figure $3 \mathrm{~d}$ ), and relatively small numbers of virus-infected cells were also observed in the perilymph (ST and SV) (Figure 3e). Viral infection was not observed in the scala media $(\mathrm{SM})$ in the endolymph, including the Corti organ (CO) (Figure 3b-e), in accordance with the findings reported previously. ${ }^{17}$ However, virus-infected cells were observed in the spiral limbus (SL), including the stria vascularis (SV), in two cases (Figure 3f).

In the i.p.-infected mice with i.c. injection of LPS, the number of virus-infected cells was high in the mesenchymal regions, moderate in $\mathrm{CN}$ regions, and low in the perilymph. No virus-infected cells were observed in the endolymph (Figure 4a).

The distribution of virus-infected cells in the brains was examined in the cases in which virus-infected cells were observed in the inner ears. Clusters of virus-infected cells were observed in the ventricular walls, including the subventricular zone (SVZ) (Figure 3g). Virus-infected cells were also observed in the meninges (Men) (Figure 3h). There was a correlation between the detection of virus-infected cells in the perilymph in the inner ear and detection of virus-infected cells in the meninges in the brain. These regions are connected anatomically by the cochlear aqueduct. ${ }^{18}$

\section{Comparison of MCMV Infection in the Labyrinths Between i.p.-Infected Mice and i.c.-Infected Mice}

Infection (i.c.) with MCMV resulted in the spread of infection into the inner ears in most of the mice without injection of LPS (Figure 5). Virus-infected cells were predominantly observed in epithelial cells of the perilymph, including ST and $\mathrm{SV}$ (Figures $4 \mathrm{~b}$ and 5). However, virus infection was not observed in SM in the endolymph and the accompanying SL (Figures $4 \mathrm{~b}$, and $5 \mathrm{~b}$ and c). Virus-infected cells were also prominently observed along the $\mathrm{CN}$, and were often associated with meningeal infection (Figure $5 b$ and $d$ ). In contrast, the relative number of virus-infected cells in the mesenchymal region in labyrinths was low compared with that in the i.p.-infected mice with i.c. injection of LPS (Figure $4 \mathrm{~b}$ ).

\section{Characterization of Virus-Infected Cells in the Labyrinths in MCMV-i.p.-Infected Mice with LPS}

In MCMV-i.p.-infected mice with i.c. injection of LPS, inflammatory cells, including polymorphonuclear and mononuclear cells, were infiltrated into the mesenchymal regions of the labyrinths, especially around the cochlear modiolar vessels, where inflammatory cells were prominently detected by immunostaining for MPO (Figure 6a). However, MPO-positive cells were hardly observed in the labyrinths of i.c.-infected mice (not shown). Although virus-infected cells occasionally observed in the mesenchymal regions were infiltrated with MPO-positive cells, no virus-infected cells were double-stained with anti-MPO pAb. Viral antigen-positive cells were sometimes observed in endothelial cells of these vessels (Figure 6b, arrows). In the SG, virus-infected cells were detected in non-neuronal cells, but were not detected in ganglion cells that were identified by immunostaining for NeuN (Figure 6c).

\section{Hearing Loss Induced in MCMV-Infected Mice with i.c. Injection of LPS}

The baseline hearing thresholds measured using the ABR were determined in mock- and MCMV-i.p.-infected mice 4 weeks after infection (Figure 7). In mock-infected mice with PBS or LPS injection and MCMV-i.p.-infected mice with PBS injection, the mean SPL was less than $35 \mathrm{~dB}$. Only MCMVi.p.-infected mice with i.c. injection of LPS showed a significant increase in the mean SPL, though the intensity of SPL in mice varied from 45 to $85 \mathrm{~dB}$ (Supplementary Table 1).

\section{DISCUSSION}

SNHL is the most frequent sequela of congenital CMV infection. ${ }^{26} \mathrm{~A}$ retrospective study revealed that about $10 \%$ of infants with congenital hearing loss and $35 \%$ of those with moderate to severe late-onset hearing loss were infected with CMV at birth. ${ }^{11,27}$ In Japan, in about $15 \%$ of cases of SNHL, CMV was detected in the DNA specimens prepared from dried umbilical cords, which are available for every child born in Japan. ${ }^{12}$ Furthermore, CMV DNA was directly detected in clinical samples of perilymph from patients with deafness due to congenital CMV infection by real-time PCR. ${ }^{28}$

The most interesting question is why the inner ears are so vulnerable after congenital CMV infection. To address this question, animal models of hearing loss caused by CMV infection in the inner ears have been devised. In guinea-pig models, there have been many reports in which CMV was directly inoculated into the inner ears or perilymph by sur- 

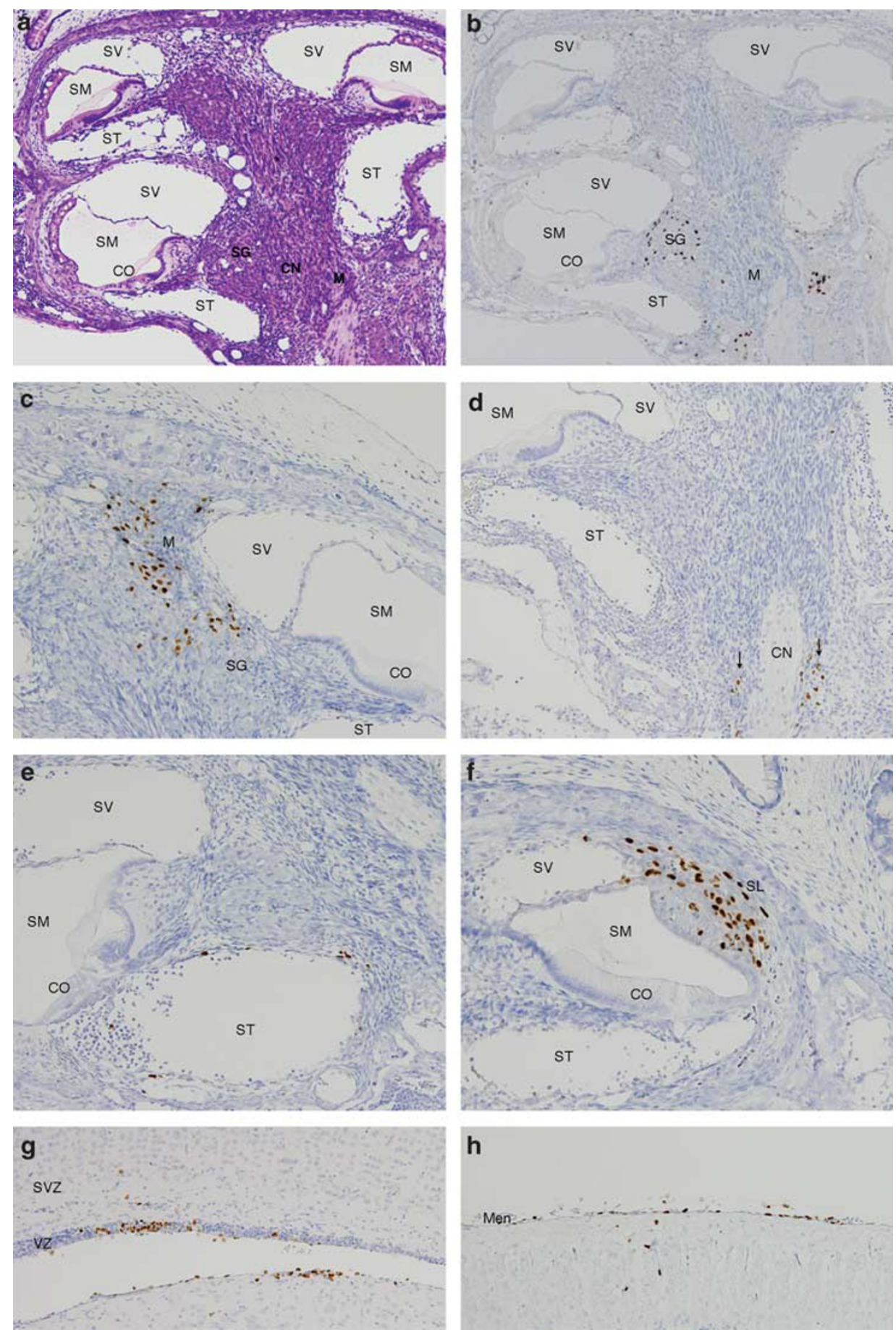

Figure 3 Localization of MCMV IE1-positive cells in the labyrinths in the MCMV-i.p.-infected mice with i.c. injections of LPS. Newborn mice were i.p. infected with MCMV and received i.c. injection of LPS four times. Mice were killed 6 days after infection, and serial sections of the inner ears were made after fixation and decalcification. Sections were reacted with anti-IE1 mAb, incubated with horseradish peroxidase-labeled secondary Ab, and colored with DAB.

(a) Low-power view of the labyrinth, HE stain. CN, cochlear nerve; M, mesenchymal region; SG, spiral ganglia; ST, scala tympani; SV, scala vestibuli; SM, scala media; CO, Corti organ. (b) IE1-positive cells in the adjacent section. IE1-positive cells were observed in the following higher power views: (c) in the mesenchymal region, (d) along the cochlear nerve (arrows), (e) in the epithelial cells of ST, (f) in the spiral limbus (SL), (g) in the cerebral ventricular wall. VZ, ventricular zone; SVZ, subventricular zone and (h) in the cerebral meninges (Men).

gical operations in young adult or adult animals. ${ }^{13,15,16}$ In newborn mice with i.c. infection with MCMV, infection of the inner ears occurred in a high proportion of animals with a large number of infected cells, as reported by Davis and Hawrisiak. ${ }^{18}$ Labyrinthitis was also reported after systemic infection of young adult guinea-pigs with $\mathrm{GPCMV}^{29}$ and in 
a

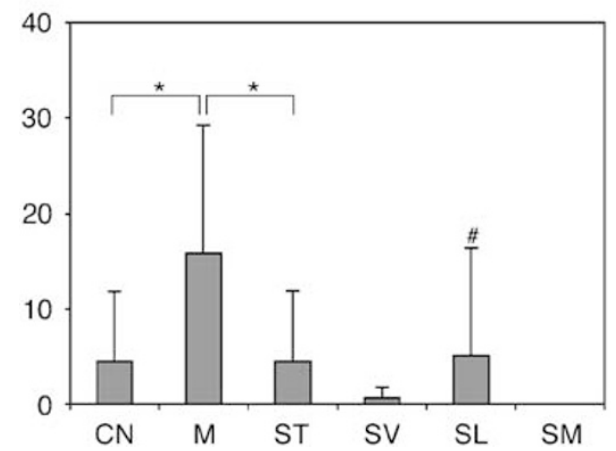

b

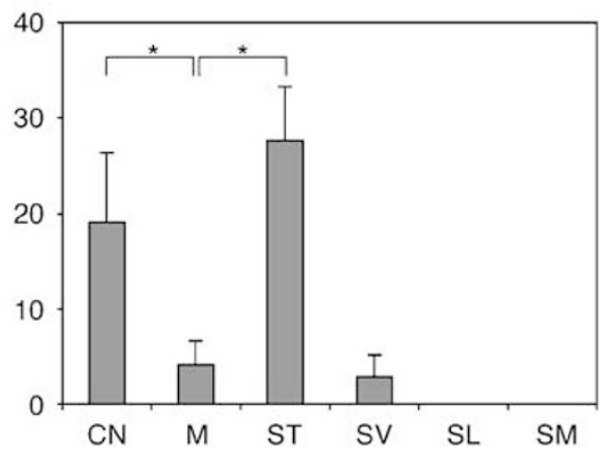

Figure 4 Distribution of IE1-positive cells in the labyrinths in the MCMV-i.p.-infected mice with i.c. injections of LPS (a) and in the MCMV-i.c.-infected mice (b). In both groups, newborn mice were infected and killed 6 days after infection. After immunohistochemical staining, IE1-positive cells were counted in the portions of the labyrinths, such as cochlear nerve (CN), mesenchymal (M) region, including spiral ganglia (SG), scala tympani (ST), scala vestibuli (SV), spiral limbus (SL) and scala media (SM). The number of IE1-positive cells was expressed as the average number \pm s.d. per labyrinth. Virus-infected cells were counted in three sections per each animal and at least eight animals were assessed. ${ }^{\star} P<0.05$ by Student's $t$-test. ${ }^{*}$ Only two cases had positive cells in this lesion.

guinea-pig fetuses after vertical transmission by infection of pregnant mothers with GPCMV. ${ }^{17}$ In the present study, we have established an experimental model of hearing loss in mice systemically infected by i.p. inoculation of MCMV into newborn mice. Systemic infection of mice at this stage is similar to congenital CMV infection in humans, since the newborn mouse ear is developmentally immature and embryologically equivalent to a human ear of 15 weeks of gestation. ${ }^{30}$

We found that susceptibility of the inner ears to MCMV infection was markedly increased by four times i.c. injection of LPS. Since systemic administration of LPS had a fatal effect on MCMV-infected newborn mice, we tried to perform i.c. injection repeatedly to mice in a sublethal condition. In experimental retinitis achieved by intraocular MCMV infection, viral replication within the eye occurred when mice were rendered in an immunosuppressive condition by systemic irradiation and administration of antilymphocyte serum. ${ }^{31}$ It was also reported that viral replication in the retina was induced after systemic infection with MCMV by local or systemic injection of LPS in young adult mice. ${ }^{32}$

CMV infection in the inner ear is considered to occur through three routes: (a) meningogenic route along the $\mathrm{CN}$, (b) the tympanogenic route through the cochlear aqueduct, and (c) the hematogenous route. ${ }^{15,33}$ In the MCMV-i.c.-mice, a large number of virus-infected cells were observed in the perilymph and along the $\mathrm{CN}$ due to meningogenic expansion, but virus infection did not extend into the endolymph, including $\mathrm{CO}$, as reported previously. ${ }^{17,29}$ In the brains of MCMV-i.c.-infected mice, infection spread into the inner ears from the meninges and ventricles, which are connected to the perilymph through the cochlear aqueduct, ${ }^{33}$ as reported by Davis and Hawrisiak. ${ }^{18}$ It is possible that the endolymph is protected from tympanogenic and meningogenic infection with MCMV by the bloodlabyrinth barrier (BLB). ${ }^{34}$
In systemic infection in mice established by i.p. inoculation with MCMV, injection of LPS into the brain induced viral infection in the inner ear, mainly in the mesenchymal regions, in which cochlear modiolar vessels are prominent near the cochlear ducts. These vessels and mesenchymal cells seem to be target structures for CMV infection in the inner ears. Interestingly, mesenchymal cells in the mouse inner ear are derived from bone marrow hematopoietic stem cells. ${ }^{35}$ Although no virus-infected cells were observed in MPO-positive cells in the present study, it was reported that hematogenic CMV infection tends to occur via infected hematopoietic progenitor cells. ${ }^{36}$ It was reported that the modiolar vessels were infected in the inner ears of adult guinea-pigs by systemic infection with intracardial inoculation of GPCMV, but no infection was detected within the $\mathrm{SV}^{29}$ In our study, virus infection was also observed in the epithelial cells of mesenchymal regions and the SL, including $\mathrm{SV}$, which is the site of the BLB, ${ }^{34}$ although infection did not extend into the endolymphs, including the CO. Infection of these regions was not observed by i.c. infection, as described above. These findings suggested that LPS enhances hematogenous spread into the inner ears in systemic infection, resulting in the possibility of disruption of the BLB.

In systemic infection, injection of LPS also stimulated the brain infection, especially in the ventricular walls, including the VZ and SVZ, and also in the meninges, where bloodcerebrospinal fluid (CSF) barriers are located. ${ }^{37}$ This stimulation of brain infection is probably caused by disruption of the BBB and blood-CSF barrier by LPS, resulting in infection from the brain into the inner ears, probably through meningogenic and tympanogenic routes, as described in the i.c.-infected brains where infection had already passed through the bloodCSF barrier. ${ }^{18}$ Therefore, a small or moderate number of virusinfected cells were also observed in the $\mathrm{CN}$ and the perilymph in the i.p.-infected mice stimulated by LPS. 

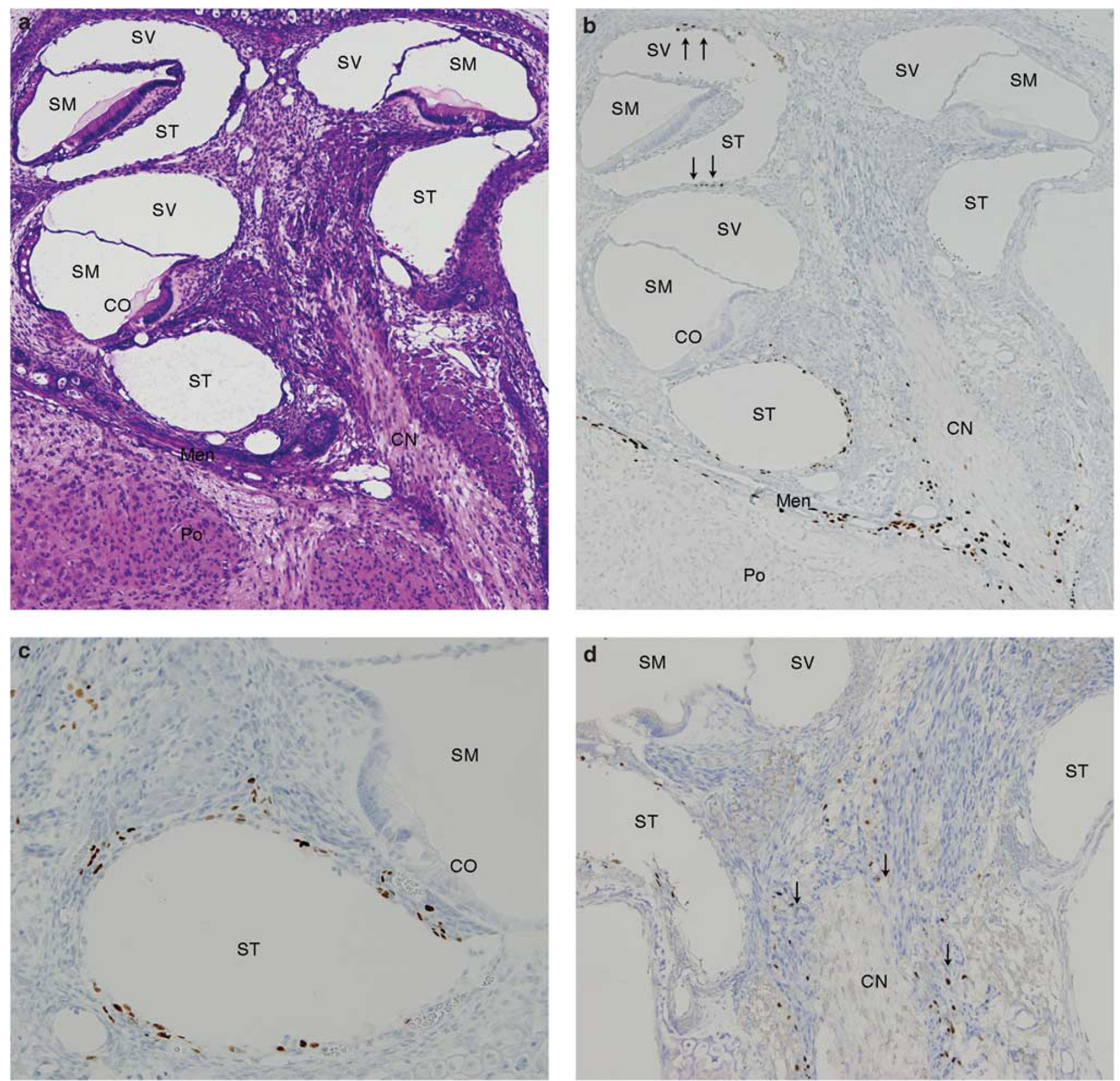

Figure 5 Localization of IE1-positive cells in the labyrinths in the MCMV-i.c.-infected mice. Newborn mice were i.c. infected with MCMV and killed 6 days after infection. (a) Low-power view of labyrinth, HE stain. SV, scala vestibuli; (b) IE1-positive cells in the adjacent section. Men, meninges; Po, pons. (c) IE1positive cells in scala tympani (ST), and lack of infected cells in scala media (SM), including Corti organ (CO). (d) IE1-positive cells along the cochlear nerve (CN).

It is noteworthy that LPS injection induced a conspicuous number of inflammatory cells in the mesenchymal region, especially around the cochlear modiolar vessels, where inflammatory cells were prominently detected with MPO staining. Virus-infected cells were associated with these inflammatory responses. Therefore, it is possible that LPSinduced inflammation stimulates MCMV replication in the labyrinths. Similarly, LPS induced MCMV replication in the retina ${ }^{32}$ and in the myocardia of mice. ${ }^{38}$ Dissemination of CMV in the blood occurs mainly via leukocytes and these cells are major source of CMV spread. ${ }^{39}$ Penetration of blood cells into the labyrinths may be facilitated by disruption of the BLB by LPS like the blood-retinal barrier. ${ }^{32}$ Endothelial activation by LPS may also stimulate CMV replication. ${ }^{40}$

The receptors of the innate immune system are activated by microbial components such as LPS, which is a key molecule involved in the initiation of the sepsis syndrome. ${ }^{41,42}$ Miller et al. ${ }^{43}$ proposed the hypothesis that the variability of bacterial ligands, such as LPS and their innate 

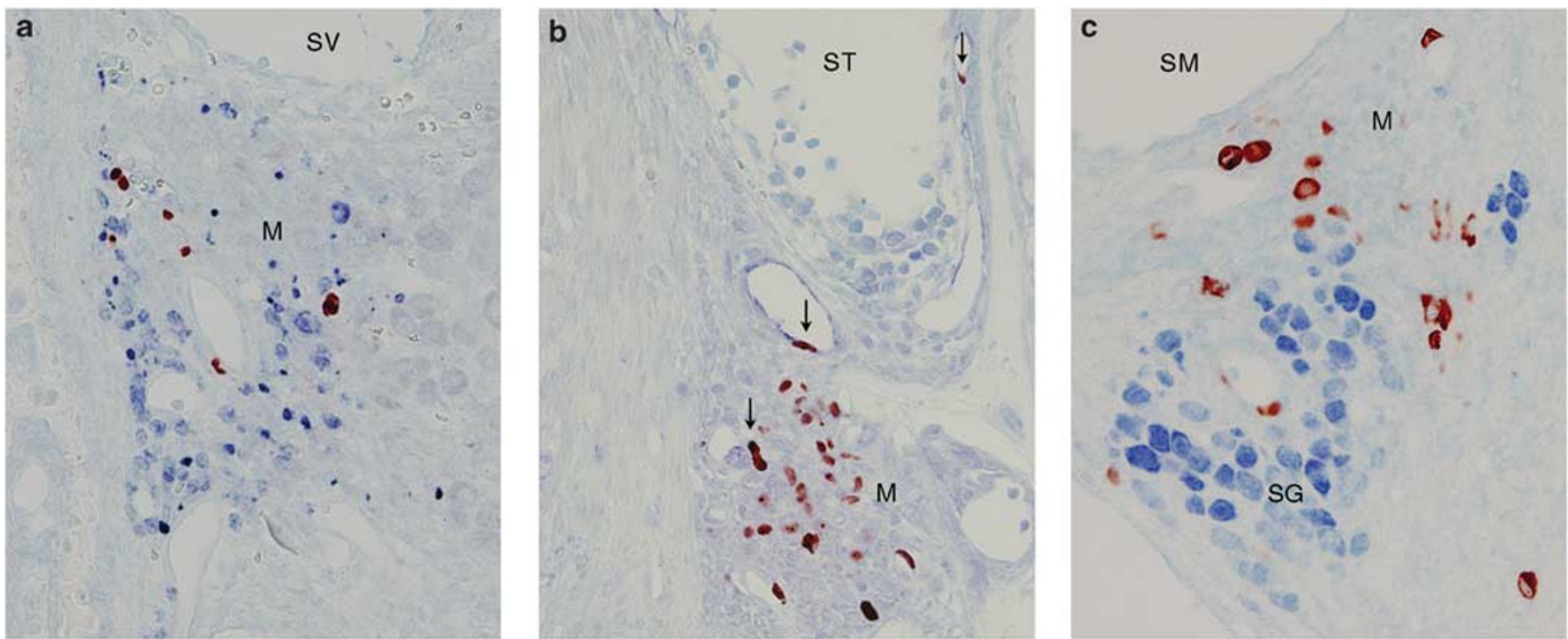

Figure 6 Immunohistochemical double staining of the labyrinths with antibodies against the viral antigen and antibodies specific to myeloperoxidase (MPO) (a), factor VIII (b), or neuronal nuclei (NeuN) (c) in the MCMV-i.p.-infected mice with LPS. (a) Sections were reacted with anti-IE1 mAb, incubated with secondary $\mathrm{Ab}$, and colored with AEC, then reacted with anti-MPO pAb and incubated with ALP-conjugated secondary Ab, colored with Fast Blue. M, mesenchymal region; SV, scala vestibuli. (b) Sections were reacted with anti-IE1 mAb, incubated with secondary Ab, and colored with AEC, then reacted with anti-factor VIII pAb and incubated with ALP-conjugated secondary Ab and colored with Fast Blue. Arrows show double-stained cells. ST, scala tympani. (c) Sections were reacted with anti-Q3 (cytoplasmic antigen) $\mathrm{mAb}$, incubated with secondary Ab, and colored with AEC, then sections were reacted with anti-NeuN mAb and incubated with ALP-conjugated secondary Ab and colored with Fast Blue. SG, spiral ganglia; SM, scala media.
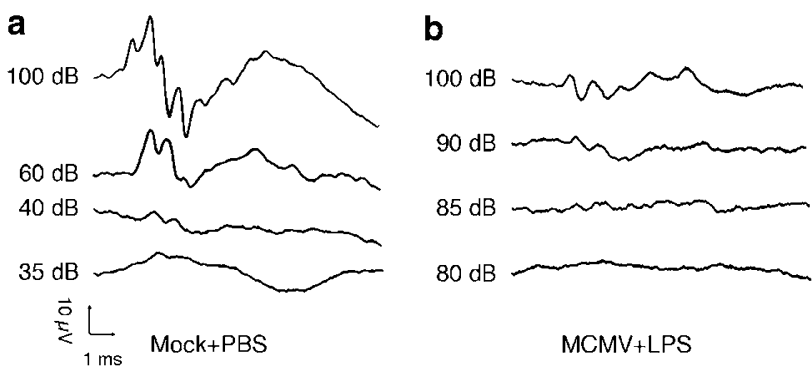

Figure 7 Typical profiles of auditory brainstem response (ABR) of mockinfected and MCMV-i.p.-infected mice with i.c. injection of LPS 4 weeks after infection. In mock-infected mouse (a) ABR disappeared at $35 \mathrm{~dB} S \mathrm{SP}$, while in MCMV-i.p.-infected mouse with i.c. injections of LPS (b) ABR disappeared at $80 \mathrm{~dB}$ SPL and the amplitude of ABR was lower than that in control mouse at any SPL.

immune receptors, is an important factor in determining the outcome of infectious disease. There is a relationship between the clinical course of patients with septic shock and acute CMV infection. ${ }^{44}$ Pereira et al..$^{45}$ reported that CMV infection depends on the presence of other pathogens, such as bacteria, and on the coordinated immune response to viral replication in the placenta. LPS is known to signal via Toll-like receptor (TLR)-4, thus having pro-inflammatory properties. ${ }^{42}$ Therefore, septic systemic inflammation caused by bacterial infection in patients with congenital CMV infection may enhance susceptibility of the inner ear to CMV infection, as in the inner ears of MCMV-infected mice stimulated by LPS. Furthermore, it has been reported that bacterial infection or administration of LPS triggers reactivation of latent pulmonary CMV in immunocompetent mice. ${ }^{46}$ The fact that mannitol, another reagent for disruption of the BBB, did not induce viral antigen-positive cells in the inner ears suggests that only certain types of TLR ligands such as LPS may facilitate CMV-induced hearing loss.

Recently, Schraff et al. ${ }^{47}$ reported that inner ear inflammation triggered by CMV-coding pro-inflammatory protein may play a role in CMV-related auditory pathogenesis in the guinea-pig model. Similarly, we hypothesize that inflammation elicited by bacterial infection enhances CMVrelated auditory pathogenesis in the patients with congenital CMV infection.

In conclusion, LPS enhanced the susceptibility of the inner ear to MCMV replication after systemic infection of newborn mice. Mesenchymal vessels around the labyrinth ducts seem to be the most susceptible target for hematogenous infection of the inner ear, in addition to meningogenic and tympanogenic spread of infection from the infected brain. Since LPS-elicited inflammation in the labyrinths stimulated viral replication, systemic bacterial infection such as that occurs in sepsis may be the trigger for hearing loss after congenital CMV infection in humans.

Supplementary Information accompanies the paper on the Laboratory Investigation website (http://www.laboratoryinvestigation.org)

\section{ACKNOWLEDGEMENTS}

We thank Mr Masaaki Kaneta in our laboratory for his excellent technical assistance. We also thank Dr Stipan Jonjic, Department of Histology and Embryology, University of Rijeka, Croatia, for mouse anti-IE1 mAb. This study 
was supported in part by a grant (no. 15390126) from the Ministry of Education, Culture, Science, and Technology, in Japan.

1. Stagno S, Pass RF, Cloud G, et al. Primary cytomegalovirus infection in pregnancy. Incidence, transmission to fetus, and clinical outcome. JAMA 1986;256:1904-1908.

2. Demmler GJ. Infectious Diseases Society of America and Centers for Disease Control. Summary of a workshop on surveillance for congenital cytomegalovirus disease. Rev Infect Dis 1991;13:315-329.

3. Bale Jr JF, Bray PF, Bell WE. Neuroradiographic abnormalities in congenital cytomegalovirus infection. Pediatr Neurol 1985;1:42-47.

4. Cinque $P$, Marenzi $R$, Ceresa $D$. Cytomegalovirus infections of the nervous system. Intervirology 1997;40:85-97.

5. Pass RF, Stagno S, Myers GJ, et al. Outcome of symptomatic congenital cytomegalovirus infection: results of long-term longitudinal follow-up. Pediatrics 1980;66:758-762.

6. Conboy TJ, Pass RF, Stagno S, et al. Intellectual development in schoolaged children with asymptomatic congenital cytomegalovirus infection. Pediatrics 1986;77:801-806.

7. Rivera LB, Boppana SB, Fowler KB, et al. Predictors of hearing loss in children with symptomatic congenital cytomegalovirus infection. Pediatrics 2002;110:762-767.

8. Ross SA, Fowler KB, Ashrith G, et al. Hearing loss in children with congenital cytomegalovirus infection born to mothers with preexisting immunity. J Pediatr 2006;148:332-336.

9. Dahle AJ, Fowler KB, Wright JD, et al. Longitudinal investigation of hearing disorders in children with congenital cytomegalovirus. J Am Acad Audiol 2000;11:283-290.

10. Fowler KB, Boppana SB. Congenital cytomegalovirus (CMV) infection and hearing deficit. J Clin Virol 2006;35:226-231.

11. Morton CC, Nance WE. Newborn hearing screening-a silent revolution. N Engl J Med 2006;354:2151-2164.

12. Ogawa H, Suzutani $T, B a b a Y$, et al. Etiology of severe sensorineural hearing loss in children: independent impact of congenital cytomegalovirus infection and GJB2 mutations. J Infect Dis 2007; 195:782-788.

13. Harris JP, Woolf NK, Ryan AF, et al. Immunologic and electrophysiological response to cytomegaloviral inner ear infection in the guinea pig. J Infect Dis 1984;150:523-530.

14. Keithley EM, Sharp P, Woolf NK, et al. Temporal sequence of viral antigen expression in the cochlea induced by cytomegalovirus. Acta Otolaryngol 1988;106:46-54.

15. Nomura Y, Hara M, Kurata T. Experimental herpes simplex virus and cytomegalovirus labyrinthitis. Acta Otolaryngol 1988;457(Suppl):57-66.

16. White DR, Choo DI, Stroup G, et al. The effect of cidofovir on cytomegalovirus-induced hearing loss in a guinea pig model. Arch Otolaryngol Head Neck Surg 2006;132:608-615.

17. Katano H, Sato Y, Tsutsui Y, et al. Pathogenesis of cytomegalovirusassociated labyrinthitis in a guinea pig model. Microbes Infect 2007;9:183-191.

18. Davis GL, Hawrisiak MM. Experimental cytomegalovirus infection and the developing mouse inner ear: in vivo and in vitro studies. Lab Invest 1977;37:20-29.

19. Li R-Y, Tsutsui Y. Growth retardation and microcephaly induced in mice by placental infection with murine cytomegalovirus. Teratology 2000;62:79-85.

20. Tsutsui $Y$, Kosugi I, Kawasaki H. Neuropathogenesis in cytomegalovirus infection: indication of the mechanisms using mouse models. Rev Med Virol 2005;15:327-345.

21. Tsutsui $Y$, Kashiwai A, Kawamura N, et al. Prolonged infection of mouse brain neurons with murine cytomegalovirus after pre- and perinatal infection. Arch Virol 1995;140:1725-1736.

22. Wentworth BB, French L. Plaque assay of cytomegalovirus strains of human origin. Proc Soc Exp Bio Med 1970;135:253-258.

23. Cosolo WC, Martinello P, Louis WJ, et al. Blood-brain barrier disruption using mannitol: time course and electron microscopy studies. Am J Physiol 1989;256(2 Part 2):R443-R447.

24. Kosugi I, Kawasaki H, Arai $\mathrm{Y}$, et al. Innate immune responses to cytomegalovirus infection in the developing mouse brain and their evasion by virus-infected neurons. Am J Pathol 2002;161:919-928.
25. Shinmura Y, Aiba-Masago S, Kosugi I, et al. Differential expression of the immediate-early and early antigens in neuronal and glia cells of developing mouse brains infected with murine cytomegalovirus. Am J Pathol 1997;151:1331-1340.

26. Pass RF. Congenital cytomegalovirus infection and hearing loss. Herpes 2005; 12:50-55.

27. Barbi M, Binda S, Caroppo S, et al. A wide role for congenital cytomegalovirus infection in sensorineural hearing loss. Pediatr Infect Dis 2003;22:39-42.

28. Sugiura S, Yoshikawa T, Nishiyama Y, et al. Detection of human cytomegalovirus DNA in perilymph of patients with sensorineural hearing loss using real-time PCR. J Med Virol 2003;69:72-75.

29. Fukuda S, Keithley EM, Harris JP. Experimental cytomegalovirus infection: viremic spread to the inner ear. Am J Otolaryngol 1988;9:135-141.

30. Otis E, Brent R. Equivalent ages in mouse and human embryos. Anat Rec 1954;120:33.

31. Gao EK, Yu XH, Lin CP, et al. Intraocular viral replication after systemic murine cytomegalovirus infection requires immunosuppression. Invest Ophthalmol Vis Sci 1995;36:2322-2327.

32. Zhang M, Xin H, Atherton SS. Murine cytomegalovirus (MCMV) spreads to and replicates in the retina after endotoxin-induced disruption of the blood-retinal barrier of immunosuppressed BALB/c mice. J Neurovirol 2005;11:365-375.

33. Kastenbauer $S$, Klein $M$, Koedel $U$, et al. Reactive nitrogen species contribute to blood-labyrinth barrier disruption in suppurative labyrinthitis complicating experimental pneumococcal meningitis in the rat. Brain Res 2001;904:208-217.

34. Juhn SK, Hunter BA, Odland RM. Blood-labyrinth barrier and fluid dynamics of the inner ear. Int Tinnitus J 2001;7:72-83.

35. Lang $\mathrm{H}$, Ebihara $\mathrm{Y}, \mathrm{Schmiedt}$ RA, et al. Contribution of bone marrow hematopoietic stem cells to adult mouse inner ear: mesenchymal cells and fibrocytes. J Comp Neurol 2006;496:187-201.

36. Noda S, Aguirre SA, Bitmansour A, et al. Cytomegalovirus MCK-2 controls mobilization and recruitment of myeloid progenitor cells to facilitate dissemination. Blood 2006;107:30-38.

37. Saunders NR, Habgood MD, Dziegielewska KM. Barrier mechanisms in the brain, II. Immature brain. Clin Exp Pharmaco Physiol 1999;26:85-91.

38. Lenzo JC, Fairweather D, Shellam GR, et al. Immunomodulation of murine cytomegalovirus-induced myocarditis in mice treated with lipopolysaccharide and tumor necrosis factor. Cell Immunol 2001;213:52-61.

39. Taylor-Wiedeman J, Sissons JG, Borysiewicz LK, et al. Monocytes are a major site of persistence of human cytomegalovirus in peripheral blood mononuclear cells. J Gen Virol 1991;72: 2059-2064.

40. Grafte A, van der Giessen M, van Son W, et al. Circulating cytomegalovirus (CMV)-infected endothelial cells in patients with an active CMV infection. J Infect Dis 1993;167:270-277.

41. Medzhitov R. Toll-like receptors and innate immunity. Nat Rev Immunol 2001:1:135-145.

42. Dauphinee SM, Karsan A. Lipopolysaccharide signaling in endothelial cells. Lab Invest 2006;86:9-22.

43. Miller SI, Ernst RK, Bader MW. LPS, TLR4 and infectious disease diversity. Nat Rev Microbiol 2005;3:36-46.

44. Von Muller L, Klemm A, Weiss $M$, et al. Active cytomegalovirus infection in patients with sepsis. Emerg Infect Dis 2006;12: 1517-1522.

45. Pereira L, Maidji E, McDonagh S, et al. Human cytomegalovirus transmission from the uterus to the placenta correlates with the presence of pathogenic bacteria and maternal immunity. J Virol 2003;77:13301-13314.

46. Cook $\mathrm{CH}$, Trgovcich J, Zimmerman PD, et al. Lipopolysaccharide, tumor necrosis factor alpha, or interleukin-1beta triggers reactivation of latent cytomegalovirus in immunocompetent mice. J Virol 2006;80:9151-9158.

47. Schraff SA, Schleiss MR, Brown DK, et al. Macrophage inflammatory protein in cytomegalovirus-related inner ear injury. Otolaryngol Head Neck Surg 2007;137:612-618. 